\title{
Estudio ser oepidemiológico de borreliosis de Lyme en la Ciudad de México y el noreste de la República Mexicana
}

Guadalupe Gordillo-Pérez, M en $C_{\text {, }}^{(1)}$ Javier Torres, PH D, ${ }^{(1)}$ Fortino Solórzano-Santos, MD, (2) Verónica $\mathrm{G}$ arduño-Bautista, Q FB, ${ }^{(1)}$ Roberto Tapia-Conyer, $M$ en $C,{ }^{(3)} O$ nofre Muñoz, M en $C$. $^{(1)}$

\begin{abstract}
Gordillo-Pérez G,Torres J, Solórzano-Santos F, Garduño-Bautista V,Tapia-Conyer R, Muñoz 0. Estudio seroepidemiológico de borreliosis de Lyme en la Ciudad de México y el noreste de la República Mexicana. Salud Publica Mex 2003;45:351-355. El texto completo en inglés de este artículo está disponible en: http://www.insp.mx/salud/index.html
\end{abstract}

\section{Resumen}

Objetivo. Investigar mediante métodos serológicos la infección por B burgdorferi en individuos del Distrito Federal y la zona noreste de México. Material y métodos Se obtuvo una muestra representativa de sueros del Distrito Federal y la zona no reste de México, obtenidas en la Encuesta Seroepidemiológica N acional de 1987-1988. Se detectaron anticuerpos IgG vs B burgdorferi por ELISA, confirmados con Western blot. En este trabajo se utilizó estadística descriptiva. Resultados. Fueron estudiados 2346 sueros; 297 $(12.6 \%)$ fueron positivos por inmunoensayo enzimático, y 122/297 fueron confirmados por W estern blot. La seroprevalencia fue de $3.43 \%$ en el Distrito Federal y $6.2 \%$ en la zona no reste del país. Tamaulipas fue el estado con la seroprevalencia más alta. Conclusiones La prevalencia de casos seropositivos sugieren que la infección por B burgdorferi ocurre en el noreste de México y el Distrito Federal. Es necesario identificar casos clínicos y buscar el vector infectado para confirmar la presencia de la enfermedad de Lyme en México. El texto completo en inglés de este artículo está disponible en: http://www.insp.mx/salud/index.html

Palabras clave: Borrelia burgdorferi; enfermedad de Lyme; serodiagnóstico; México

\author{
Gordillo-Pérez G,Torres J, Solórzano-Santos F, \\ Garduño-BautistaV,Tapia-Conyer R, Muñoz 0. \\ Seroepidemiologic survey of Lyme Borreliosis \\ in Mexico City and the Northeast region of the country. \\ Salud Publica Mex 2003;45:351-355. \\ The English version of this paper \\ is available at: http://www.insp.mx/salud/index.html
}

\begin{abstract}
A bstract
Objective. To detect serological evidence of B burgdorferi infection in individuals from Mexico $C$ ity and from the $\mathrm{N}$ ortheast Region of the country. Material and Methods A representative sample size of serum from Mexico City and the states of the $\mathrm{N}$ ortheast of Mexico were taken from serum samples corresponding to the 1987-1988 national survey were obtained from the $\mathrm{N}$ ational Serum Bank.Antibodies against $B$ burgdorferi were detected by ELISA and confirmed with W estern blot (W B) assays. D ata were analyzed using descriptive statistics. Results. A total of 2346 serum samples were tested; 297 (12.6\%) were positive for ELISA, and 122 of 297 were confirmed by W B. Seroprevalence was $3.43 \%$ in Mexico City and $6.2 \%$ in the N ortheast region of the country. Tamaulipas was the state with the highest seroprevalence. Conclusions The prevalence of seropositive cases shows that borrelial infection is present in the northeast of Mexico and Mexico City. Identification of clinical cases and infected tick vectors is necessary to confirm the presence of Lyme disease in Mexico. The English version of this paper is available at:http://ww w.insp.mx/ salud/index.html
\end{abstract}

Key words: Borrelia burgdorferi; Lyme disease; serodiagnosis; Mexico

Este trabajo fue apoyado por el Consejo N acional de Ciencia y Tecnología de México (proyecto 30694-M).

(1) Unidad de Investigación Médica en Enfermedades Infecciosas y Parasitarias. Centro Médico N acional Siglo XXI (C MN SXXI). Instituto Mexicano del Seguro Social (IMSS), México, DF, México.

(2) Departamento de Infectología, Hospital de Pediatría, CMN SXXI, IMSS, México, DF, México.

(3) Subsecretaría de Salud, México, DF, México.

Fecha de recibido: 28 de octubre de 2002 • Fecha de aprobado: 23 de abril de 2003

Solicitud de sobretiros: M en C. María Guadalupe Gordillo Pérez. Unidad de Investigación Médica en Enfermedades Infecciosas, Hospital de Pediatría, Centro Médico N acional Siglo XXI, IMSS.Avenida Cuauhtémoc 330, colonia D octores 06725 México, DF, México.

Correo electrónico: lugope2@ hotmail.com.mx 
L a enfermedad de Lyme o borreliosis de Lyme es una enfermedad infecciosa emergente causada por la espiroqueta Borrelia burgdorferi. Esta bacteria es transmitida por la mordedura de las garrapatas del género Ixodes. En los Estados Unidos de América (EUA) y en Europa, la borreliosis de Lyme representa 90\% de las infecciones transmitidas por garrapatas. ${ }^{1}$

La enfermedad es endémica en la mayoría de los países europeos, en tres zonas enzoóticas de EUA y también en algunos países asiáticos. En Europa la prevalencia de la infección evaluada con pruebas de inmunoensayo enzimático (ELISA) o inmunofluorescencia (IFA) es de 8 a $27 \%{ }^{2}$ y en EUA varía de 1 a $10 \% .^{3}$ En Suramérica se han reportado casos clínicos sugestivos de la enfermedad, aunque sin confirmación por estudios de laboratorio. ${ }^{4}$

En México se ha identificado el vector Ixodes en Baja California, la Península de Yucatán, el Golfo de México y la zona noreste de la República. ${ }^{5}$ Algunos casos sugestivos de borreliosis de Lyme fueron descritos a principios de la década de 1990 en los estados de Sinaloa y Nuevo León, sin que se haya logrado la confirmación etiológica. ${ }^{6,7}$ En 1999, en un estudio de seroprevalencia epidemiológica nacional, se encontró positividad por ELISA en $1.1 \%$ de las muestras y se confirmó ésta en $0.3 \%$ de las mismas mediante inmunotransferencia. Los sujetos seropositivos fueron ubicados en la zona noreste y centro de la República Mexicana. ${ }^{8}$ Considerando la posible existencia de casos clínicos sugestivos de borreliosis de Lyme en nuestro país y la presencia del vector, los reservorios y los casos seropositivos detectados en la encuesta nacional, es oportuno tener una idea acerca de la prevalencia de la infección por la bacteria causante de la enfermedad de Lyme en muestras de suero de habitantes de estas zonas.

En este estudio se determinó la prevalencia de la infección causada por Borrelia burgdorferi en una muestra de sueros de habitantes de la Ciudad de México y de la zona noreste de la República Mexicana.

\section{Material y métodos}

Estudio transversal descriptivo. El Banco Nacional de Sueros proporcionó 2346 sueros de sujetos procedentes de comunidades urbanas y rurales de los estados de Nuevo León, Tamaulipas, Coahuila y el Distrito Federal (DF). Se seleccionaron al azar los sueros de sujetos de 1 a 90 años de edad de uno y otro sexo y de diferentes niveles socioeconómicos. ${ }^{9}$ Se calcularon porcentajes.

Estudios serológicos. Se realizó un inmunoensayo enzimático directo (ELISA) con antígenos de espiroquetas completas de la cepa CN40 de B burgdorferi (antígeno donado por el doctor Magnarelli, New Haven, Connecticut, EUA). El procedimiento se realizó de acuerdo con lo descrito por Rusell, ${ }^{10}$ con algunas modificaciones reportadas en este trabajo; ${ }^{8}$ se aplicó el antígeno $(\mathrm{Ag})$, a una concentración de $5 \mu \mathrm{g} / \mathrm{ml}$ por pozo, en microplacas de 96 pozos; el suero se diluyó a 1:640 y se usó como conjugado el anti-IgG humano de cabra, unida a fosfatasa alcalina (Biosource, CA, EUA) diluido 1:1000. El valor de corte se obtuvo con el promedio de 26 sueros de sujetos mexicanos no infectados con $B$ burgdorferi ( 15 sueros de menores de edad y 11 de adultos sanos) \pm 3 desviaciones estándar. ${ }^{8}$ Como control positivo se usó una mezcla de cinco sueros de pacientes positivos de una zona endémica del noreste de EUA (sueros donados por el doctor Magnarelli). Con estos datos se construyó una curva de regresión lineal que se probó durante cinco días diferentes, obteniéndose así una correlación de $97.8 \%$ al repetir los ensayos. Un suero se consideró como positivo cuando la absorbancia fue igual o mayor al valor del punto de corte más tres desviaciones estándar. ${ }^{10}$

El control positivo fue incluido por cuadruplicado en cada microplaca. Cada muestra de suero fue probada por duplicado, y las muestras positivas fueron tituladas desde 1:640 a 1:10280.

Como un método comprobatorio de la serorreactividad específica, las muestras positivas por ELISA fueron probadas por el método de inmunotransferencia (Western-Blot) ${ }^{11}$ usando como antígeno la cepa CN40 de B burgdorferi. Se corrieron $200 \mu \mathrm{g}$ de Ag en minigeles de poliacrilamida a $12 \%$; el suero problema se probó a una dilución de 1:50. El mismo conjugado descrito se utilizó para el ELISA y su sustrato fue NBT-BCIP (GibcoBRL Gaistherburg, EUA). Asimismo, en cada membrana se incluyeron los sueros testigo positivos y negativos, además de los sueros de pacientes con lupus eritematoso sistémico (LES), sífilis, artritis reumatoide y anticuerpos antifosfolípidos. Se consideró un suero positivo a $B$ burgdorferi ss (Bbss) si había cuando menos cinco de las siguientes bandas de proteínas: $18,23,28,30,39,41,45,58,66$, y $93 \mathrm{kDa}$, de acuerdo con los criterios del Centro de Control de Enfermedades de Atlanta $(C D C)^{12}$ Con los criterios de la Unión Europea contra la enfermedad de Lyme (EUCALB) se detectaron como mínimo tres de las siguientes bandas de proteínas de $B b s s$ p18, 21, 23, 27, 31, 34, 39, 41, 66, 75 o $93 \mathrm{kDa} \cdot{ }^{13,14}$

Para validar este ensayo de inmunotransferencia se seleccionaron al azar 10\% de los sueros positivos por ELISA y se probaron por inmunotransferencia con un equipo comercial (MarDx Diagnostics, Carlsbad, CA) el cual contiene los antígenos de B burgdorferi cepa 
B31, cuyos pesos moleculares están bien definidos. Este equipo comercial fue validado por el CDC y tiene una especificidad mayor a $98 \% .^{15}$

\section{Resultados}

Se estudió un total de 2346 sueros de personas de la zona noreste de México y del DF. Por ELISA se detectaron 297 sueros con anticuerpos IgG vs B burgdorferi ss, lo cual corresponde a una prevalencia de $7.28 \%$ en el DF y $15 \%$ en la zona noreste (cuadro I). La mediana de la edad de los sujetos positivos fue de 34 años y la relación masculino: femenino fue de 1.6:1. Sin embargo, en el estado de Coahuila se obtuvo una mediana de 14 años y un intervalo intercuartílico (25 a 75) de 8-20 años. En las personas de la zona noreste que resultaron positivas se encontraron títulos de $\operatorname{IgG}$ por ELISA mayores a 1:2560; mientras que en el DF la mayoría de los sueros fueron positivos a títulos de 1:640.

Por el método de inmunotransferencia, en el DF fueron confirmados como seropositivos $25 / 728$ sueros $(3.43 \%)$; mientras que en la zona noreste se confirmaron $97 / 1618$ sueros (6\%). En esta zona la prevalencia confirmada para cada estado de la República Mexicana fue 3.91\% en Nuevo León, 14\% en Tamaulipas y 6\% en Coahuila (figura 1). Por otra parte, más de 50\% de los sueros de los pacientes positivos reaccionaron contra las proteínas de 30, 39, 41, 45 y 58 kDa; mientras que la reacción contra las proteínas de 18 y $23 \mathrm{Kda}$ se observó en $22 \%$ de estos sueros.

Con el equipo comercial MarDx Diagnostics de WB se confirmaron 19/20 sueros positivos (cinco del DF y 14 de la zona noreste), empleando los criterios

\section{Cuadro I \\ Prevalencia de la infección} POR Borrelia buRgdorferI DeteCtadA POR ELISA E INMUNOTRANSFERENCIA. MÉXICO, 1987-1988

\begin{tabular}{|c|c|c|c|c|}
\hline Estado & $\begin{array}{c}\text { Distrito Federal } \\
* N=728 \\
\neq m=34 a^{5}\end{array}$ & $\begin{array}{c}\text { Nuevo León } \\
N=1199 \\
m=33 a\end{array}$ & $\begin{array}{l}\text { Tamaulipas } \\
N=303 \\
m=34 a\end{array}$ & $\begin{array}{c}\text { Coahuila } \\
\mathrm{N}=116 \\
\mathrm{~m}=13.5 \mathrm{a}\end{array}$ \\
\hline Edad & \#(18.5-42.5 a) & (18-42 a) & (18.5-42.5 a) & $(7-25 a)$ \\
\hline ELISA (t) & $53(7.2 \%)$ & $141(11.7 \%)$ & $88(29 \%)$ & $15(12.9 \%)$ \\
\hline$\overline{W B}(+)$ & $25(3.4 \%)$ & $47(3.9 \%)$ & $43(14.1 \%)$ & $7(6 \%)$ \\
\hline \multicolumn{5}{|c|}{$\begin{array}{l}* \mathrm{~N}=\mathrm{N} \text { úmero de sueros } \\
\neq \mathrm{m}=\text { mediana } \\
\S \mathrm{a}=\text { años }\end{array}$} \\
\hline
\end{tabular}

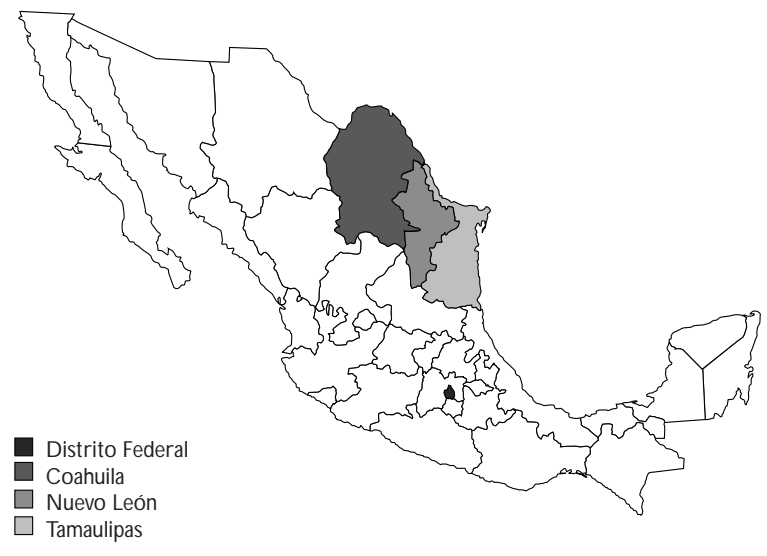

Figura 1. Prevalencia de infección por Borrelia burgdorferi en la CiUdad de México y la zona noReste de la República Mexicana

descritos por el CDC. ${ }^{12}$ Para esta prueba se obtuvo una concordancia $k=0.95$.

\section{Discusión}

Tomando en cuenta los antecedentes en el país respecto de la presencia del vector, ${ }^{5}$ huéspedes intermediarios infectados por Borrelia spp, ${ }^{16}$ casos clínicos sospecho$\operatorname{sos}^{6,7}$ y los resultados de los estudios recientes sobre prevalencia en humanos y animales ${ }^{8,16}$ que sugieren la existencia de posibles zonas endémicas de la enfermedad de Lyme, se consideró oportuno y conveniente evaluar los sueros obtenidos en la Encuesta Seroepidemiológica Nacional que correspondieran a áreas rurales y urbanas de la zona noreste del país y del DF. El tamaño de muestra necesario se calculó con base en la prevalencia obtenida en la encuesta nacional. ${ }^{8}$ De acuerdo con lo anterior los sueros en los estados de Nuevo León y Tamaulipas se probaron en 95\% de la muestra, en tanto que en Coahuila y el DF se probó en $80 \%$ de ellas.

En los estados de Nuevo León, Tamaulipas y el DF los sujetos seropositivos para $B$ burgdorferi tuvieron entre 19 y 42 años de edad, fueron mayoritariamente sujetos económicamente activos. En este grupo de personas, la infección por Borrelia burgdorferi quizá está relacionada con la exposición ocupacional. En contraste, los resultados encontrados en Coahuila muestran que la población infectada fue más joven. Predominaron adolescentes. Por lo anterior, es recomendable elaborar más estudios para demostrar si en Coahuila la exposición al vector se presenta en forma 
más temprana y, además, si existe relación entre la exposición ocupacional y la infección por Borrelia burgdorferi en otros estados. Fahrer y colaboradores en 1991 encontraron, en Europa, una curva bimodal en la edad de exposición y presentación de la enfermedad en donde la población en riesgo es mayor, entre los 10 a 14 años de edad, y de los 20 a los 40 años de edad, ${ }^{2}$ sin precisar el motivo de este comportamiento. La prevalencia encontrada por ELISA en la zona noreste (15\%), y la encontrada en la Ciudad de México (7.2\%), fue semejante a la reportada en algunas zonas endémicas de EUA y Europa. ${ }^{2,3}$

La mayoría de los estudios sobre seroprevalencia para infección por $B$ burgdorferi en la población en general, reportados en el mundo, se han hecho utilizando un método de escrutinio como el ELISA. ${ }^{2,3}$ Sin embargo, en la última década se han reconocido las limitaciones de este método, debido principalmente a la presencia de reacciones cruzadas con otras espiroquetas y enfermedades autoinmunes. Por lo anterior, el CDC recomienda la inmunotransferencia como el método para confirmar el diagnóstico serológico de infección por $B$ burgdorferi. ${ }^{12}$ Dresler ${ }^{11}$ y Tilton ${ }^{15}$ han reportado que la mitad de los sueros positivos por ELISA pueden ser confirmados con inmunotransferencia. En este estudio la inmunotransferencia confirmó $45 \%$ de los sueros positivos por ELISA. Esto sugiere que en la población mexicana existe reacción cruzada contra otros antígenos en una frecuencia similar a la de otros países.

Los criterios para considerar un WB positivo han sufrido modificaciones en EUA y Europa:;1-14 lo anterior está relacionado con la heterogeneidad genética de la bacteria y su área geográfica. Por lo ya visto, en este estudio se incluyeron los criterios establecidos por el CDC, en EUA, y la EUCALB, en Europa. Sin embargo, también se incluyeron 13 bandas, las 10 reportadas por el CDC específico para $B$ burgdorferi sensu stricto, $\mathrm{y}$ otras tres bandas presentes en $B$ burgdorferi sensu lato. Al menos dos de las bandas de las proteínas de 39, 41, 45 y $58 \mathrm{kDa}$ fueron consideradas como necesarias para confirmar el diagnóstico por Western-blot, lo cual fue encontrado en todas las muestras consideradas positivas. Dressler y colaboradores (1993) ${ }^{11}$ reportaron en los casos con enfermedad temprana la presencia de anticuerpos evidentes en las bandas de proteínas de $41 \mathrm{kDa}$ (flagelar) y $23 \mathrm{kDa}$ (OspC, proteína de membrana externa). En este estudio $10 \%$ de los casos presentó la banda de $23 \mathrm{kDa}$, detectada principalmente en las muestras de Nuevo León y Tamaulipas. En la zona noreste 3\% de los casos positivos mostraron anticuerpos contra la banda de $93 \mathrm{kDa}$, la cual es una proteína asociada con la infección tardía. ${ }^{11}$
Este estudio, por su diseño transversal con muestras séricas obtenidas hace varios años, sólo permite postular la existencia de la enfermedad de Lyme en la zona noreste del país y en la Ciudad de México. Para tener una información más certera de esta enfermedad en México se requiere la identificación y el seguimiento de aquellos pacientes que presentan manifestaciones clínicas de la enfermedad y el antecedente de mordedura de la garrapata. En estudios realizados en 1999 por nuestro grupo de trabajo se han detectado pacientes con infección neurológica y cutánea, y hay evidencias serológicas, histológicas y moleculares de probable infección por Borrelia burgdorferi. ${ }^{17}$

Los resultados descritos en este artículo intentan llamar la atención del personal clínico de salud, así como de epidemiólogos para que tomen en consideración la probable presencia de la enfermedad de Lyme en las zonas consideradas en este estudio.

\section{Referencias}

1. Steere AC. Lyme disease. N Engl J Med 1989;321:586-595 2. Fahrer $H$,Van der LSM, Sauvain MJ, Gern L, Zhioua E,A eschliman A. The prevalence and incidence of clinical and asymptomatic Lyme borreliosis in a population at risk. Infect D is 1991;163:305-310. 3. Steere AC, Taylor E, W ilson ML, Levine JF, Spielman A. Longitudinal assessment of the clinical and epidemiological features of Lyme disease in a defined population.J Infect D is 1986;154:295-300.

4. Guzmán L, N eira O. Lyme disease in Chile.J Rheumatol 1993;20: 774-775.

5. Hoffmann A. Monografía de los Ixodoidea de México. Rev Soc Mex $H$ ist $N$ at 1962;XX III:197-282.

6. Maradiaga-C eceña MA, Llausás-Vargas A, Barguera-Heredia J, Kumate-Rodríguez J. Eritema crónico migratorio asociado a artritis. Enfermedad de Lyme 0 una variante. Rev Mex Reumatol 1991;6:61. 7.Arroyave MC, Támez GR. Enfermedad de Lyme, informe de dos casos. Bol Med Hosp Infant Mex 1994;51:117-121.

8. Gordillo G, Torres J, Solórzano F, C edillo RR,Tapia CR, Muñoz 0 Serologic evidences suggesting the presence of Borrelia burgdorferi infection in Mexico. Arch Med Res 1999;30:64-68.

9. Tapia CR, G utiérrez G, Sepúlveda J. Metodología de la Encuesta N acional Seroepidemiológica, México. Salud Publica Mex 1992;34: 124-135.

10. Russell H, Sampson JS, Schmid G P,W ilkinson HW, Plikaytis B. Enzyme-linked immunosorbent assay and indirect immunofluorescence assay for Lyme disease. J Infec $D$ is 1984;149:465-470.

11. D ressler F,W halen JA, Remhardt BN, Steere AC.W estern blotting in the serodiagnosis of Lyme disease.J Infec D is 1993;167:392-400.

12. Centers for Disease Control and Prevention. Recomendations for test performance and interpretation from the second national conference on serologic diagnosis of Lyme disease. Morbid Mortal W kly Rep 1995;44:590.

13. N orman GL,Antig JM, Bigaignon G , Hogrefe W R. Serodiagnosis of Lyme borreliosis by Borrelia burgdorferi sensu stricto, B. garinii y B. afzelii western blots (immunoblots). J Clin Microbiol 1996;34:1732-1738. 
14. Hauser $U$, Lehnert $G, W$ ilske B.Validity of interpretation criteria for standarized western blots (immunoblots) for serodiagnosis of Lyme borreliosis based on sera collected throughout Europe.J C lin Microbiol 1999;37:2241-2247

15.Tilton RC, Sand MR, Manak M.The western immunoblot for Lyme disease. D etermination of sensitivity, specificity, and interpretive criteria with use of commercially available performance panels. Clin Infect D is 1997;25(Suppl 1):S31-S34.
16. Martínez A, Salinas A, Martínez F, C antú A, Miller DK. Serosurvey for selected disease agents in white-tailed deer from Mexico. J W ildlife $D$ is 1999;35(4):799- 803

17. Gordillo-Pérez MG, Estrada-Correa G, Solórzano-Santos F, Torres-López J, Muñoz HO Evidencias serológicas y moleculares de neuroborreliosis de Lyme en México. XXIII Reunión Anual de la A cademia de N eurología; 1999 oct 30-nov 2; Los Cabos, Baja California Sur, México. 